Nervenarzt 2020 $\cdot 91: 714-721$

https://doi.org/10.1007/s00115-020-00948-3

Online publiziert: 9 . Juli 2020

(c) Der/die Autor(en) 2020

\author{
Maike F. Dohrn ${ }^{1}$. Natalie Winter ${ }^{2} \cdot$ Manuel Dafotakis $^{1}$ \\ 'Neurologie, Uniklinik RWTH Aachen, Aachen, Deutschland \\ ${ }^{2}$ Neurologie, Universitätsklinikum Tübingen, Tübingen, Deutschland
}

\title{
Ursachen, Spektrum und Therapie der diabetischen Neuropathie
}

\section{Klinik}

Die Diagnose einer DN wird nach klinischen Kriterien gestellt und durch Zusatzuntersuchungen wie die Elektroneurographie unterstützt [3]. Aufgrund der hohen Prävalenz des Diabetes mellitus in der kaukasischen Allgemeinbevölkerung muss berücksichtigt werden, dass nicht jede Koinzidenz einer Polyneuropathie als DN zu werten ist [4]. Basierend auf Verteilungsmuster, Verlauf, Komorbiditäten, Sozial- und Familienanamnese müssen andere, z. T. behandelbare Erkrankungen wie die chronisch-inflammatorische demyelinisierende Polyradikulopathie (CIDP) oder die hereditäre Transthyretin-Amyloidose auch bei bestehendem Diabetes mellitus erkannt werden, da diese spezifische Therapiemöglichkeiten bedingen [3].

Die neurologische Untersuchung eines Diabetespatienten sollte immer eine Inspektion der entkleideten Extremitäten, eine detaillierte Sensibilitätstestung einschließlich Berührungs-, Vibrations- und Lageempfinden zur Erfassung großer sowie Spitz-Stumpf-Diskrimination und Temperaturempfinden zur Untersuchung kleiner Nervenfasern, eine Gangprüfung mit Frage nach afferenter Ataxie oder Steppergang, eine Einzelkraftprüfung sowie Reflextestung enthalten. Im Rahmen der Disease-Management-Programme sind auch Allgemeinmediziner und Internisten angehalten, mittels Monofilament und Vibrationsgabel $(64 \mathrm{~Hz})$ einen orientierenden Sensibilitätsstatus zu erheben [5].
DN können fokal und generalisiert sein, längenabhängig oder diffus verlaufen, überwiegend die kleinen intraepidermalen $\mathrm{C}$ - und $\mathrm{A} \delta$-Nervenfasern betreffen oder zur Ausprägung einer Polyneuropathie führen (•Abb. 1). Am häufigsten ist die distal symmetrische diabetische Polyneuropathie (DSPN), die schätzungsweise $75 \%$ aller Verläufe ausmacht [6]. Initial manifestiert sie sich mit sensiblen Defiziten der Füße. Neuropathische Schmerzen treten etwa bei $25 \%$ der Betroffenen auf. Häufig beschrieben als brennend, stechend oder „schraubstockartig“ gehen sie mit einem erheblichen Leidensdruck einher und bedürfen einer langfristigen symptomatischen Therapie [7]. Paresen finden sich bei 1-6\% aller Diabetiker [2] und betreffen vor allem die distalen Extremitäten, was die Sturzgefahr erhöht. In einer Erhebung der Mayo Clinic aus dem Jahr 1993 wurden bei etwa zwei Drittel der untersuchten Diabetiker Zeichen einer Neuropathie nachgewiesen, während nur $20 \%$ tatsächlich symptomatisch waren [2].

Autonome diabetische Neuropathien können isoliert oder, häufiger, in Kombination mit anderen DN-Formen auftreten und dabei gleichermaßen das sympathische und/oder parasympathische System betreffen. Wichtige Manifestationen sind gastrointestinale Passagestörungen, fäkale Inkontinenz oder neurogene Blasenentleerungsstörungen, Erektions- und Ejakulationsstörungen, orthostatische Hypotonie, Ruhetachykardie, Störungen des Schwitzens sowie der Pupillomotorik. Hypoglykämien werden zum Teil erst verspätet bemerkt. Die kardiovaskuläre autonome Neuropa- 


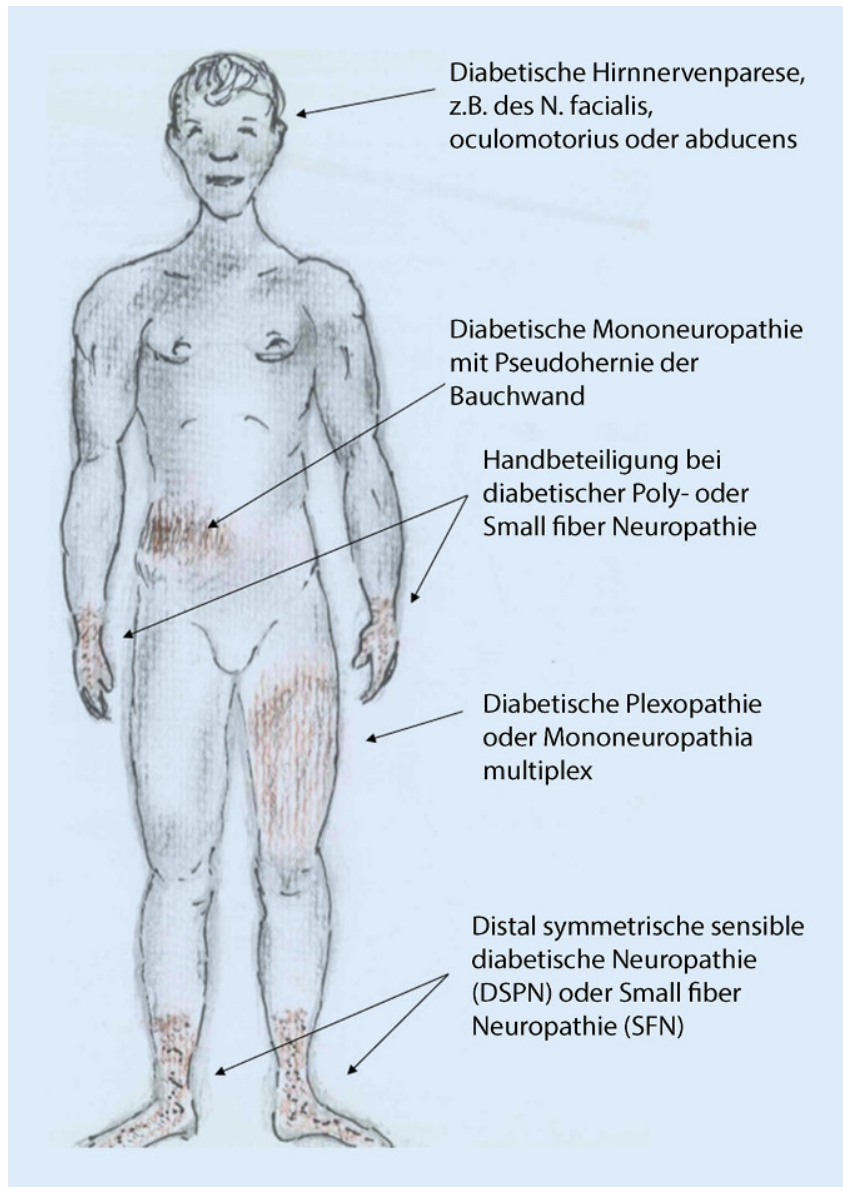

gig, durchaus aber auch diskontinuierlich sein [3]. Eine vergleichsweise akute Small fiber Neuropathie mit starken neuropathischen Schmerzen und begleitenden autonomen Symptomen kann durch zu schnelle und dabei rigorose Senkung des $\mathrm{HbA}_{1 \mathrm{c}}$-Werts $(>2 \%$ innerhalb von drei Monaten) ausgelöst werden [12]. Als Ursache werden inflammatorische Prozesse vermutet, die aber bislang nicht vollständig verstanden sind.

\section{Typ-1- und Typ-2-Diabetes mellitus: unterschiedliche Wege zur Neuropathie}

Das relative Neuropathierisiko eines Typ-1-Diabetikers kann durch optimale Glukosekontrolle um 78\% gesenkt werden. Dagegen gelingt bei Typ-2-Diabetikern durch dieselben Maßnahmen nur eine 5- bis 9\%ige Risikoreduktion [1]. Dass der Diagnosestellung eines Typ-2-Diabetes mellitus oft lange Zeiträume unerkannter Hyperglykämien vorausgehen, ist nur eine von mehreren Hypothesen, die diesen Unterschied zu erklären versucht. Da es auch im Rahmen des prädiabetischen metabolischen Syndroms zum Erwerb einer Neuropathie kommen kann, scheinen auch andere vaskuläre Risikofaktoren, insbesondere Dyslipidämie, Übergewicht, Nikotinkonsum und arterielle Hypertonie zur Pathophysiologie beizutragen $[13,14]$. Transkriptomanalysen aus Nervenbiopsien von Patienten mit Typ-2Diabetes mellitus deuten auf "pathways“ von Inflammation und Adipogenese hin, die in diesem Patientenkollektiv recht spezifisch hochreguliert sind. Polymorphismen in Genen wie $A C E, A K R 1 B 1$, APOE, NOS3, TLR4 und VEGF wirken sich potenziell begünstigend für den Erwerb einer diabetischen Polyneuropathie aus [15]. Insulin selbst, welches bei Typ-1-Diabetikern absolut, bei Typ2-Diabetikern nur relativ erniedrigt ist, könnte als neuronaler Wachstumsfaktor [16] abhängig von der Art des Mangels bzw. dem Beginn der Substitution einen direkten Einfluss auf die Entwicklung einer DN haben. 
Nervenarzt 2020 $91: 714-721$ https://doi.org/10.1007/s00115-020-00948-3

(c) Der/die Autor(en) 2020

\section{F. Dohrn · N. Winter · M. Dafotakis}

\section{Ursachen, Spektrum und Therapie der diabetischen Neuropathie}

\section{Zusammenfassung}

Hintergrund. Die Hälfte aller Diabetiker ist von einer diabetischen Neuropathie betroffen. Mikroangiopathie, dysfunktionale SchwannZell-Interaktion, Akkumulation toxischer Metabolite und inflammatorische Prozesse führen gemeinsam zur Nervenschädigung. Ziel der Arbeit. Aus- und Überblick zum aktuellen Kenntnisstand der Pathophysiologie mit aktuellen und zukünftigen Therapieimplikationen.

Methoden. Literaturrecherche (1990-2020). Ergebnisse. Klinisch führend sind sensible und autonome Symptome, Paresen können jedoch auftreten. Komplikationen wie stumme Myokardinfarkte oder das diabetische Fußsyndrom können lebensbedrohlich verlaufen und zu schwerer Behinderung führen.
In ihrer Pathophysiologie unterscheiden sich Neuropathien bei Typ-1- und Typ-2Diabetikern durch die Gegenwart zusätzlicher Risikofaktoren des metabolischen Syndroms. Die intensivierte ist der konventionellen Insulintherapie im Hinblick auf die Neuropathierisikoreduktion überlegen. Orale Antidiabetika sind nach Nebenwirkungsprofil auszuwählen. Metformin kann zu einem iatrogenen Vitamin-B12-Mangel führen. Zur Behandlung neuropathischer Schmerzen besitzt der Kalziumkanalblocker Pregabalin die höchste Empfehlungsstufe. Das Trizyklikum Amitriptylin gilt als ähnlich wirksam, ist aber bei autonomer Dysfunktion sowie kognitiven Einschränkungen kontraindiziert. Alternativ ist der Serotonin-Noradrenalin-
Wiederaufnahmehemmer Duloxetin zur symptomatischen Therapie der diabetischen Neuropathie zugelassen. Umstritten sind weitere, zum Teil nicht zugelassene Medikamente wie a-Liponsäure, Epalrestat und L-Serin. Schlussfolgerungen. Die diabetische Neuropathie ist häufig und komplikationsreich. Ein gutes Verständnis der Pathophysiologie kann zur Entwicklung neuer Therapiestrategien beitragen.

\section{Schlüsselwörter}

Typ-1- und Typ-2-Diabetes mellitus · Pathophysiologie der diabetischen Neuropathie . Neuropathische Schmerztherapie - Epalrestat . Alpha-Liponsäure

\section{Causes, spectrum, and treatment of the diabetic neuropathy}

\section{Abstract}

Background. Half of all diabetics are affected by a diabetic neuropathy. Microangiopathy, dysfunctional Schwann cell interactions, accumulation of toxic metabolites, and inflammatory processes all contribute to nerve damage.

Objective. Overview and perspectives of the pathophysiology as well as the current and future treatment implications.

Methods. Literature search (1990-2020). Results. Clinically predominant are sensory and autonomic symptoms; however, muscle weakness can occur as well. Complications such as unrecognized myocardial infarctions and the diabetic foot syndrome are potentially life-threatening and can cause major disability. The pathophysiology of neuropathies in type 1 and type 2 diabetes mellitus differs due to additional risk factors of the metabolic syndrome. To reduce the risk of neuropathy, an intensive insulin therapy is superior compared to the conventional insulin therapy. Oral antidiabetic drugs should be chosen based on individual risk profiles. Metformin can cause an iatrogenic vitamin B12 deficiency. In the treatment of neuropathic pain, the calcium channel blocker pregabalin has the highest recommendation level. The tricyclic antidepressant amitriptyline is considered to be equally effective, but it is contraindicated in autonomic dysregulation and cognitive impairment. Alternatively, the serotoninnorepinephrine reuptake inhibitor duloxetine is approved for the symptomatic treatment of diabetic neuropathies. Controversially discussed medications include alpha-lipoic acid, epalrestat, and L-serine.

Conclusion. The diabetic neuropathy is frequent and causes severe complications. A good understanding of the underlying pathophysiology can contribute to the development of novel treatment strategies in the future.

\section{Keywords}

Type 1 and type 2 diabetes mellitus . Pathophysiology of diabetic neuropathies . Neuropathic pain therapy · Epalrestat · Alpha lipoic acid

\section{Pathophysiologie}

Ursachen und Risiken für das Entstehen einer DN sind vielfältig. Wir werden sie im Folgenden schematisch vereinfacht zusammenfassen (• Abb. 2).

\section{- Akkumulation toxischer Metabolite:}

Bei hyperglykämischer Stoffwechsellage akkumuliert Glukose in Nerven-, Nieren- und Retinazellen, wo es unter Verbrauch von NADPH zur Bildung von Sorbitol kommt. Als Zwischenstufe in der Umwandlung von Glukose zu Fruktose und um- gekehrt ist Sorbitol physiologischer Bestandteil des Polyolstoffwechsels. Als zellschädigend wird neben der osmotischen Wirkung auch die durch die verminderte Verfügbarkeit von NADPH vermehrte Anfälligkeit für oxidativen Stress diskutiert [17]. Die Aldosereduktase ist das geschwindigkeitsbestimmende Enzym der Polyolbildung. Zu einer Enzymüberexpression führende Polymorphismen im AKR1B1-Gen kommen in ca. $26 \%$ der Bevölkerung vor und sind mit einem höheren Risi- ko vergesellschaftet, bei vorliegendem Diabetes eine Neuropathie zu entwickeln. Im Mai 2020 wurde erstmalig eine erbliche axonale Neuropathie beschrieben, die durch biallelische Mutationen im SORD-Gen ähnlich der diabetischen Neuropathie mit Störungen im Sorbitolstoffwechsel assoziiert ist [18].

Alanin ist eine glukogene Aminosäure, die bei Diabetikern erhöhte Serumspiegel aufweist. Hierdurch wird die Serin-Palmitoyl-CoA-Transferase, das geschwindigkeitsbestimmen- 




Abb. $2 \triangle$ Pathophysiologie der DN als Schemazeichnung

de Enzym der Sphingolipidsynthese, zum Substratwechsel verleitet, sodass die für den Abbau der Sphingoidbasen entscheidende $\mathrm{OH}$-Gruppe fehlt [19]. Desoxysphingolipide führen zu einem verminderten axonalen Aussprossen und sind bei Patienten mit diabetischer Polyneuropathie erhöht [19].

- Inflammatorische Prozesse: Durch Bildung von ,advanced glycation end-products" (AGE) und mitochondriale Dysfunktion entsteht oxidativer Stress, sodass es zu DNASchäden und somit zu Zellnekrosen oder, vermittelt durch Zytokine wie z. B. TNF- $\alpha$ und IL-6, zur Inflammation kommt [20]. Inflammatorische Prozesse werden durch den Transkriptionsfaktor NF- $\kappa B$ verstärkt, der über den Phospholipase-C-Pathway exprimiert und in den Zellkern verlagert wird. In Gegenwart ungefalteter Proteine oder Störungen der Kalziumhomöostase reagiert das endoplasmatische Retikulum überlastet („ER-Stress“), was wiederum zur Bildung freier Radikale sowie zur Induktion proinflammatorischer Kaskaden führt. Im Mausmodell konnten in Korrelation zum ERStress erniedrigte sensomotorische Nervenantwortpotenziale sowie eine Reduktion der intraepidermalen Nervenfaserdichte nachgewiesen werden [21].

- Dysfunktionale Schwann-Zell-Interaktion: Axone sind aufgrund ihrer Länge und der damit assoziierten Entfernung vom Zellkörper besonders vulnerabel für eine Schädigung und in ihrem Metabolismus auf den engen Kontakt zu Schwann-Zellen angewiesen. Über Gap junctions werden sie mit Metaboliten wie Laktat versorgt [22], ein Mechanismus, der sich bei systemischer Insulinresistenz erschöpft. In Zellkulturen kommt es unter Laktatdeprivation zu einer verminderten Neurofilamentexpression und, hier diskutiert für das zentrale Nervensystem, zum Verlust von Axonen [22]. Besonders vulnerabel für eine Minderversorgung mit ATP erscheinen dünn oder unmyelinisierte Axone, weil die nichtsaltatorische Leitung vergleichsweise energieaufwändiger ist [23]. Eine Lipidüberladung von Schwann-Zellen führt darüber hinaus zur Bildung neuro- toxischer Acylcarnitine, welche bei Weitergabe an die Axone zu einer Störung der Mitochondrienfunktion führen [24].

- Mikroangiopathie: Vaskuläre Risikofaktoren wie Hypercholesterinämie, Hypertonie und Übergewicht sind unabhängig von der Glukosekontrolle mit einer höheren Auftretenswahrscheinlichkeit einer DN assoziiert [13], und auch bei prädiabetischem metabolischem Syndrom kann eine DN entstehen. Derselbe oxidative Stress, der auf Nervenzellen einwirkt, erreicht auch das vaskuläre Kompartiment. Mikrovaskuläre Veränderungen äußern sich bereits früh durch eine insuffiziente NO-mediierte Vasodilatation mit Auffälligkeiten von Blutfluss und Gefäßpermeabilität [25]. Es kommt zur Endotheldysfunktion und zur Überproliferation der Extrazellulärmatrix [25]. Als Ausdruck einer endoneuronalen Mikroangiopathie [26] konnten sowohl bei Patienten mit diabetischer als auch mit prädiabetischer Neuropathie verdickte Basalmembranen mit endothelialer Hyperplasie sowie verminderte endoluminale Durchmesser 
Tab. 1 Medikamentöse Behandlungskonzepte der diabetischen Neuropathie. In fetter Schrift dargestellt sind Medikamentenklassen, die zur Blutzuckerkontrolle eingesetzt werden, in kursiver Schrift Medikamente, die zur Therapie neuropathischer Schmerzen empfohlen werden. In normaler

Schrift dargestellt werden beispielhaft Medikamente aufgeführt, die in die Pathophysiologie der DN eingreifen, deren Nutzen aber so unzureichend belegt ist, dass es bisher zu keiner weltweiten Zulassung gekommen ist

\begin{tabular}{|c|c|c|c|c|c|}
\hline $\begin{array}{l}\text { Substanz- } \\
\text { klasse }\end{array}$ & Wirkziel & Wirkstoffbeispiele & Indikation & Kontraindikationen & Nebenwirkungen \\
\hline \multirow[t]{2}{*}{ Insulin } & \multirow[t]{2}{*}{$\begin{array}{l}\text { Blutzucker- } \\
\text { kontrolle }\end{array}$} & $\begin{array}{l}\text { Langzeitinsuline (z. B. } \\
\text { Glargin, Detemir) }\end{array}$ & \multirow[t]{2}{*}{ Typ-1/2-DM } & \multirow[t]{2}{*}{ Hypokaliämie, Anaphylaxie } & \multirow{2}{*}{$\begin{array}{l}\text { Hypoglykämie, Hypokaliämie, } \\
\text { Gewichtszunahme, } \\
\text { Lipodystrophie }\end{array}$} \\
\hline & & $\begin{array}{l}\text { Kurz wirksame Insuli- } \\
\text { ne (z. B. Lispro, Aspart) }\end{array}$ & & & \\
\hline \multirow[t]{8}{*}{$\begin{array}{l}\text { Orale Anti- } \\
\text { diabetika }\end{array}$} & \multirow[t]{8}{*}{$\begin{array}{l}\text { Blutzucker- } \\
\text { kontrolle }\end{array}$} & $\begin{array}{l}\text { Biguanide (z. B. Met- } \\
\text { formin) }\end{array}$ & \multirow[t]{8}{*}{ Typ-2-DM } & $\begin{array}{l}\text { Herz-, Leber-, Niereninsuffizienz, Alko- } \\
\text { holmissbrauch, binnen } 48 \text { h nach Gabe } \\
\text { iodhaltiger Kontrastmittel, mitochon- } \\
\text { driale Erkrankungen }\end{array}$ & $\begin{array}{l}\text { Geschmacksveränderungen, } \\
\text { gastrointestinale Beschwerden, } \\
\text { Hautreaktionen, Laktatazidose, } \\
\text { Vitamin-B12-Mangel }\end{array}$ \\
\hline & & $\begin{array}{l}\text { Sulfonylharnstoffe } \\
\text { (z. B. Glibenclamid, } \\
\text { Glimepirid) }\end{array}$ & & $\begin{array}{l}\text { Nieren- oder Leberinsuffizienz, schwe- } \\
\text { re kardiovaskuläre Komorbidität }\end{array}$ & $\begin{array}{l}\text { Hypoglykämien, Gewichtszu- } \\
\text { nahme, Blutbildveränderungen }\end{array}$ \\
\hline & & $\begin{array}{l}\text { Glinide (z. B. Nategli- } \\
\text { nid, Repaglinid) }\end{array}$ & & $\begin{array}{l}\text { Nieren- oder Leberinsuffizienz, schwe- } \\
\text { re kardiovaskuläre Komorbidität }\end{array}$ & $\begin{array}{l}\text { Hypoglykämien, Gewichtszu- } \\
\text { nahme, Hepatotoxizität }\end{array}$ \\
\hline & & $\begin{array}{l}\text { Glitazone (z. B. Piogli- } \\
\text { tazon) }\end{array}$ & & $\begin{array}{l}\text { Herz- oder Leberinsuffizienz, Uro- } \\
\text { thelkarzinom }\end{array}$ & $\begin{array}{l}\text { Gewichtszunahme, Ödeme, } \\
\text { Herzinsuffizienz, Osteoporose }\end{array}$ \\
\hline & & $\begin{array}{l}\text { DPP4-Inhibitoren (z. B. } \\
\text { Saxagliptin, Sitaglip- } \\
\text { tin) }\end{array}$ & & $\begin{array}{l}\text { Leber- oder Niereninsuffizienz, Pan- } \\
\text { kreatitis }\end{array}$ & $\begin{array}{l}\text { Gastrointestinale Beschwer- } \\
\text { den, Pankreatitis, Schwindel, } \\
\text { Kopfschmerzen }\end{array}$ \\
\hline & & $\begin{array}{l}\text { GLP1-Analoga (z. B. } \\
\text { Liraglutid, Exenatid) }\end{array}$ & & $\begin{array}{l}\text { Leber- oder Niereninsuffizienz, Pan- } \\
\text { kreatitis, Pankreas- oder medulläres } \\
\text { Schilddrüsenkarzinom, gastrointesti- } \\
\text { nale Motilitätsstörungen }\end{array}$ & $\begin{array}{l}\text { Gastrointestinale Beschwerden, } \\
\text { Pankreatitis }\end{array}$ \\
\hline & & $\begin{array}{l}\text { SGLT2-Inhibitoren } \\
\text { (z.B. Dapagliflozin) }\end{array}$ & & $\begin{array}{l}\text { Niereninsuffizienz, rezidivierende } \\
\text { Harnwegsinfektionen }\end{array}$ & $\begin{array}{l}\text { Genitalmykosen, Harnwegsin- } \\
\text { fekte, Polyurie, Ketoazidose }\end{array}$ \\
\hline & & $\begin{array}{l}\text { a-Glukosidase-Hem- } \\
\text { mer (z. B. Acarbose) }\end{array}$ & & $\begin{array}{l}\text { Niereninsuffizienz, vorbestehende } \\
\text { gastrointestinale Passagestörung }\end{array}$ & Gastrointestinale Beschwerden \\
\hline \multirow[t]{2}{*}{$\begin{array}{l}\text { Anti- } \\
\text { konvulsiva }\end{array}$} & \multirow[t]{2}{*}{$\begin{array}{l}\text { Therapie neu- } \\
\text { ropathischer } \\
\text { Schmerzen }\end{array}$} & $\begin{array}{l}\text { Kalziumkanalblocker } \\
\text { (z. B. Pregabalin, Gaba- } \\
\text { pentin) }\end{array}$ & \multirow[t]{2}{*}{$\begin{array}{l}\text { Neuropath. } \\
\text { Schmerzen }\end{array}$} & $\begin{array}{l}\text { Niereninsuffizienz, Überempfindlichkeits- } \\
\text { reaktionen }\end{array}$ & $\begin{array}{l}\text { Müdigkeit, Benommenheit, Ver- } \\
\text { schwommensehen, Depression }\end{array}$ \\
\hline & & $\begin{array}{l}\text { Natriumkanalblocker } \\
\text { (z. B. Carbamazepin) }\end{array}$ & & $\begin{array}{l}\text { Leberinsuffizienz, Porphyrie, Knochen- } \\
\text { markdepletion, Überempfindlichkeit }\end{array}$ & $\begin{array}{l}\text { Müdigkeit, UV-Empfindlichkeit, Hy- } \\
\text { ponatriämie, Cytochrominduktion }\end{array}$ \\
\hline \multirow[t]{2}{*}{$\begin{array}{l}\text { Anti- } \\
\text { depressiva }\end{array}$} & \multirow[t]{2}{*}{$\begin{array}{l}\text { Therapieneu- } \\
\text { ropathischer } \\
\text { Schmerzen }\end{array}$} & $\begin{array}{l}\text { Zyklische Antidepres- } \\
\text { siva (z. B. Amitriptylin, } \\
\text { Clomipramin) }\end{array}$ & \multirow[t]{2}{*}{$\begin{array}{l}\text { Neuropath. } \\
\text { Schmerzen }\end{array}$} & $\begin{array}{l}\text { Autonome Neuropathie, AV-Block, Leber- } \\
\text { insuffizienz, MAO-Inhibition }\end{array}$ & $\begin{array}{l}\text { Miktionsstörungen, Glaukom, } \\
\text { Hypotension, Mundtrockenheit }\end{array}$ \\
\hline & & $\begin{array}{l}\text { Selektive Serotonin-Nor- } \\
\text { adrenalin-Wiederauf- } \\
\text { nahmehemmer (z. B. } \\
\text { Duloxetin) }\end{array}$ & & $\begin{array}{l}\text { Leber- oder Niereninsuffizienz, MAO-Inhi- } \\
\text { bition, Kombination z. B. mit Fluorchinolo- } \\
\text { nen }\end{array}$ & $\begin{array}{l}\text { Übelkeit, Hypoglykämie, hyperten- } \\
\text { sive Krisen }\end{array}$ \\
\hline $\begin{array}{l}\text { a-Lipon- } \\
\text { säure }\end{array}$ & $\begin{array}{l}\text { Reduktion von } \\
\text { oxidativem } \\
\text { Stress }\end{array}$ & Thioctsäure & $\begin{array}{l}\text { DN mit } \\
\text { neuropath. } \\
\text { Schmerzen }^{\text {a }}\end{array}$ & Allergie/Unverträglichkeit & $\begin{array}{l}\text { Infusionsreaktionen, Hypogly- } \\
\text { kämien, Insulinautoimmunsyn- } \\
\text { drom, Inaktivierung von Cisplatin }\end{array}$ \\
\hline $\begin{array}{l}\text { Aldosere- } \\
\text { duktase- } \\
\text { inhibitoren }\end{array}$ & $\begin{array}{l}\text { Reduktion von } \\
\text { osmotischem } \\
\text { Stress }\end{array}$ & Epalrestat & $\mathrm{DN}^{\mathrm{b}}$ & Leberinsuffizienz & Gastrointestinale Beschwerden \\
\hline L-Serin & $\begin{array}{l}\text { Reduktion } \\
\text { toxischer } \\
\text { Sphingolipid- } \\
\text { basen }\end{array}$ & L-Serin & $-{ }^{c}$ & - & Übelkeit, Nierensteine \\
\hline \multicolumn{6}{|c|}{ 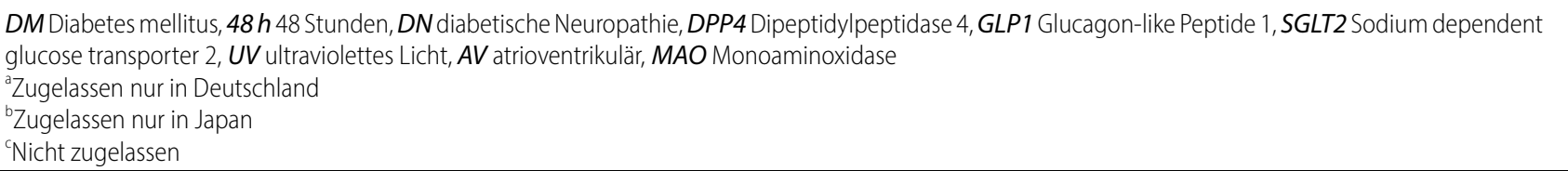 } \\
\hline
\end{tabular}


nachgewiesen werden [25-27]. Eine bedeutende ischämische Komponente als Ursache der Nervenschädigung konnte spezifisch für die diabetische Plexusneuropathie gezeigt werden, welche histologisch auf eine lokalisierte aseptische Mikrovaskulitis zurückgeführt werden konnte [11].

\section{Therapie}

\section{Blutzuckermanagement}

Essenzielle Behandlungsgrundlage der DN ist eine strikte Blutzuckereinstellung (• Tab. 1). Da aber auch Hypoglykämien zu einer axonalen Schädigung führen $[12,27]$, sind größere Blutzuckerschwankungen zu vermeiden. Die von 1982 bis 1993 an 1441 Typ-1-Diabetikern durchgeführte Studie „Diabetes Control and Complications Trial“ (DCCT) zeigte eine Risikoreduktion für den Erwerb einer Neuropathie unter intensivierter gegenüber der konventionellen Insulintherapie [28]. Dass gerade zu Beginn der Diabeteserkrankung eine bestmögliche Blutzuckerkontrolle entscheidend ist, konnte in der weitere 20 Jahre umfassenden Folgestudie „Epidemiology of Diabetes Interventions and Complications" (EDIC) gezeigt werden [29]. Verglichen mit Typ-1-Diabetikern sprechen Patienten mit Insulinresistenz und metabolischem Syndrom schlechter auf blutzuckersenkende Therapien an. Einen guten Überblick über die entsprechende Studienlage bis 2012 liefern Callaghan und Kollegen [14].

Vergleicht man orale Antidiabetika untereinander, so scheinen Insulinsensitizer gegenüber Sulfonylharnstoffen mit einem besseren Neuropathieoutcome assoziiert zu sein. Mögliche Erklärungen hierfür sind die vergleichsweise geringere Gewichtszunahme sowie die Vermeidung von Hypoglykämien. Die langfristige Einnahme von Metformin ist mit einem schwereren Neuropathieverlauf assoziiert, was auf einen iatrogenen Vitamin-B12-Mangel zurückgeführt wird [30]. Es empfiehlt sich, VitaminB12-Spiegel unter Metformintherapie zu überwachen und ggf. zu substituieren. Der Einsatz von Statinen zur Behandlung der bei metabolischem Syndrom häu- fig auftretenden Hyperlipidämie nimmt nach aktueller Studienlage keinen negativen Einfluss auf den Verlauf einer diabetischen Neuropathie [31].

\section{Symptomatische Therapie}

Wichtige Säulen der symptomatischen Therapie sind die neuropathische Schmerztherapie, Physiotherapie sowie Fußpflege und Ulkusprophylaxe.

Nach aktuellen nationalen und internationalen Leitlinien werden Medikamente aus dem Formenkreis sowohl der Antikonvulsiva als auch der Antidepressiva zur Behandlung neuropathischer Schmerzen empfohlen (• Tab. 1) [32], die ggf. kombiniert werden können. Von allen symptomatischen Therapieoptionen verfügt Pregabalin über die umfangreichste Studienlage und wird als effektiv eingestuft [14, 32]. Andere Optionen mit vergleichbarer „number needed to treat "von drei bis sechs Patienten für eine $50 \%$ ige Schmerzreduktion [33] sind Gabapentin, ebenfalls ein Kalziumkanalblocker, oder der SerotoninNoradrenalin-Wiederaufnahmehemmer Duloxetin und das Trizyklikum Amitriptylin, welche nach Ansprechen und Nebenwirkungsspektrum ausgewählt werden. Pregabalin und Gabapentin müssen bei begleitender Niereninsuffizienz in der Dosis angepasst werden. Eine häufige Nebenwirkung ist Müdigkeit. Das zyklische Antidepressivum Amitriptylin erfordert aufgrund anticholinerger Nebenwirkungen z.B. mit Mundtrockenheit eine langsame Dosissteigerung. Ein AV-Block muss im 12-Kanal-EKG ausgeschlossen werden. Bei Hinweisen auf eine begleitende autonome Neuropathie sollte auf zyklische Antidepressiva verzichtet werden. Cytochrominteraktionen sind bei beiden genannten Antidepressiva möglich. Hierfür besonders bekannt ist zudem der Natriumkanalblocker Carbamazepin aus der Gruppe der Antikonvulsiva. Es kann darüber hinaus zu Leberwerterhöhung, Hyponatriämie und Blutbildveränderungen führen, erhöht die UVEmpfindlichkeit der Haut und beeinflusst den hepatischen Abbau anderer Medikamente wie Phenprocoumon oder oraler Kontrazeptiva. Opioidanalgetika können ergänzend zur Schmerztherapie hinzugezogen werden. Ebenfalls hilfreich ist die topische Applikation von Lidocain oder Capsaicin [32].

Ziel der Schmerztherapie ist u.a. die Fähigkeit, körperlich aktiv zu sein und z. B. Physiotherapie wahrzunehmen. Dies reduziert vaskuläre Komplikationen, verbessert die Muskelkraft und verhindert Stürze. Zur Vermeidung nichtschmerzhafter Wunden müssen die Füße regelmäßig inspiziert und das Schuhwerk angepasst werden [34]. Eine gute Patientenschulung ist diesbezüglich essenziell.

\section{Weitere (experimentelle) Therapieansätze}

Aufgrund ihrer Eigenschaft als Antioxidans und Radikalfänger, Recycler von Vitamin C, E und Glutathion sowie Regulator z. B. der Expression des Transkriptionsfaktors NF- $\mathrm{KB}$ wird $\alpha-L i$ ponsäure seit Langem als potenzielles Therapeutikum diabetischer Neuropathien diskutiert. Eine vier randomisierte Studien umfassende Metaanalyse beschrieb eine signifikante Verbesserung des totalen Symptomscores sowie des Neuropathy Impairment Score der unteren Extremität nach dreiwöchiger intravenöser Gabe [35]. In Deutschland ist $\alpha$-Liponsäure zur Behandlung neuropathischer Schmerzen bei diabetischer Polyneuropathie in intravenöser Verabreichung zugelassen.

Aldosereduktaseinhibitoren setzen am Polyol-Pathway an und verhindern die Bildung von Sorbitol aus Glukose. Nach einer an 196 Patienten mit diabetischer Neuropathie durchgeführten randomisierten und placebokontrollierten Studie zu Epalrestat, welches über 12 Wochen in einer Dosierung von $150 \mathrm{mg} / \mathrm{Tag}$ oral gegeben wurde, fand in Japan die Zulassung dieses Aldosereduktaseinhibitors statt. Er hatte sich positiv auf neuropathische Schmerzen, Vibrationsempfinden und Nervenleitgeschwindigkeiten ausgewirkt [36]. Eine 10 Studien umfassenden Metaanalyse zeigte, dass sich unter Epalrestat für autonome Testparameter wie die Herzfrequenzvariabilität und den Schellong-Test marginal signifikante Besserungen bewirken ließen [36]. 
Die orale Supplementation der Aminosäure $L$-Serin kann der Bildung neurotoxischer 1-Desoxy-Sphingolipide entgegenwirken, die bei Überangebot der glukogenen Aminosäure L-Alanin entstehen [22]. An mit Streptozotocin behandelten Ratten konnten durch Zufütterung mit L-Serin eine Reduktion der 1-Desoxy-Sphingolipid-Spiegel und eine Besserung verschiedener Neuropathiekennwerte wie der thermischen „response“, der Nervenleitgeschwindigkeit und der intraepidermalen Nervenfaserdichte gezeigt werden [22]. Eine Zulassung des Nahrungsergänzungsmittels L-Serin zur Behandlung der DN ist derzeit nicht in Aussicht.

\section{Schlussfolgerungen}

Die diabetische Neuropathie ist häufig, ihr Erscheinungsbild vielfältig und komplikationsreich. Sie gilt als Ausschlussdiagnose, sodass auch bei Diabetikern an andere behandelbare Neuropathieursachen gedacht werden muss. Während sich das Risiko einer diabetischen Neuropathie bei Typ-1-Diabetikern durch Optimierung des Blutzuckers erheblich senken lässt, nehmen beim Typ2-Diabetes andere Faktoren des metabolischen Syndroms ebenfalls Einfluss auf die insgesamt schlechtere Prognose. Symptomatische Therapien neuropathischer Schmerzen stammen aus dem Formenkreis der Antikonvulsiva und Antidepressiva. Gerade bei autonomer Beteiligung ist auf etwaige anticholinerge Nebenwirkungen zu achten. Die Entwicklung von Ursachentherapien bedarf weiterer Forschung.

\section{Fazit für die Praxis}

- Auch wenn die Hälfte aller Diabetiker an einer diabetischen Neuropathie erkrankt, müssen bei Koinzidenz von Diabetes mellitus und Neuropathie andere Ursachen untersucht und ggf. behandelt werden.

- Die Pathophysiologie der diabetischen Neuropathie ist komplex und ergibt sich aus einem Zusammenspiel von Mikroangiopathie, dysfunktionaler Schwann-Zell-Interaktion,
Akkumulation toxischer Metabolite und inflammatorischen Prozessen.

- Patienten mit Neuropathie bei Typ-2Diabetes mellitus sprechen schlechter auf eine therapeutische Blutzuckerkontrolle an, was vermutlich auf die begleitenden Risikofaktoren im Rahmen des metabolischen Syndroms zurückzuführen ist.

- Neuropathische Schmerzen erzeugen einen erheblichen Leidensdruck und sollten abhängig vom individuellen Nebenwirkungs- und Risikoprofil in erster Wahl mit Medikamenten wie Pregabalin oder Gabapentin, Amitriptylin oder Duloxetin behandelt werden.

- Wirkstoffe wie a-Liponsäure, Epalrestat oder L-Serin setzen an pathophysiologischen Mechanismen wie oxidativem und osmotischem Stress an, sind aber aufgrund der unzureichend nachgewiesenen Wirksamkeit nur in einzelnen Ländern bzw. gar nicht zugelassen.

\section{Korrespondenzadresse}

\section{Dr. Maike F. Dohrn}

Neurologie, Uniklinik RWTH Aachen

Pauwelsstr. 30, Aachen, Deutschland mdohrn@ukaachen.de

Danksagung. Wir bedanken uns bei Jutta Schmitz und Jorge Rato für den Entwurf der Abbildungen.

Funding. Open Access funding provided by Projekt DEAL.

\section{Einhaltung ethischer Richtlinien}

Interessenkonflikt. M.F. Dohrn, N. Winter und M. Dafotakis geben an, dass kein Interessenkonflikt besteht.

Für diesen Beitrag wurden von den Autoren keine Studien an Menschen oder Tieren durchgeführt. Für die aufgeführten Studien gelten die jeweils dort angegebenen ethischen Richtlinien.

Open Access. Dieser Artikel wird unter der Creative Commons Namensnennung 4.0 International Lizenz veröffentlicht, welche die Nutzung, Vervielfältigung, Bearbeitung, Verbreitung und Wiedergabe in jeglichem Medium und Format erlaubt, sofern Sie den/die ursprünglichen Autor(en) und die Quelle ordnungsgemäß nennen, einen Link zur Creative Commons Lizenz beifügen und angeben, ob Änderungen vorgenommen wurden.

Die in diesem Artikel enthaltenen Bilder und sonstiges Drittmaterial unterliegen ebenfalls der genannten
Creative Commons Lizenz, sofern sich aus der Abbildungslegende nichts anderes ergibt. Sofern das betreffende Material nicht unter der genannten Creative Commons Lizenz steht und die betreffende Handlung nicht nach gesetzlichen Vorschriften erlaubt ist, ist für die oben aufgeführten Weiterverwendungen des Materials die Einwilligung des jeweiligen Rechteinhabers einzuholen.

Weitere Details zur Lizenz entnehmen Sie bitte der Lizenzinformation auf http://creativecommons.org/ licenses/by/4.0/deed.de.

\section{Literatur}

1. Pop-Busui R, Boulton AJ, Feldman EL et al (2017) Diabetic neuropathy: a position statement by the American Diabetes Association. Diabetes Care 40(1):136-154

2. Dyck PJ, Kratz KM, Karnes JL et al (1993) The prevalence by staged severity of various types of diabetic neuropathy, retinopathy, and nephropathy in a population-based cohort The Rochester Diabetic Neuropathy Study. Neurology 43(4):817-817

3. Sommer C, Geber C, Young P, Forst R, Birklein F, Schoser B (2018) Polyneuropathies. Dtsch Arztebl Int 115:83-90

4. Khan C, Abholz HH, Ellger B et al (2012) Nationale VersorgungsLeitlinie Neuropathie bei Diabetes im Erwachsenenalter. Diabetol und Stoffwechsel 7(04):243-285

5. Sämann A, Tajiyeva O, Müller N et al (2008) Prevalence of the diabetic foot syndrome at the primary care level in Germany: a cross-sectional study. Diabet Med 25(5):557-563

6. Dyck PJ, Albers JW, Andersen Het al (2011) Toronto Expert Panel on Diabetic Neuropathy. Diabetic polyneuropathies: update on research definition, diagnostic criteria and estimation of severity. Diabetes Metab Res Rev 27:620-628

7. O'Connor AB (2009) Neuropathic pain: quality-of life impact, costs and cost effectiveness of therapy. Pharmacoeconomics 27:95-112

8. Maser RE, Mitchell BD, Vinik Al, Freeman R (2003) The association between cardiovascular autonomic neuropathy and mortality in individuals with diabetes: a meta-analysis. Diabetes Care 26:1895-1901

9. Albers JW, Jacobson RD, Smyth DL (2020) Diabetic amyotrophy: from the basics to the bedside. EMJ 5(1):94-103

10. Massie R, Mauermann ML, Staff NP, Amrami KK, Mandrekar JN, Dyck PJ, Klein CJ, Dyck PJB (2012) Diabetic cervical radiculoplexus neuropathy: a distinct syndrome expanding the spectrum of diabetic radiculoplexus neuropathies. Brain 135:3074-3088

11. Dyck PJB, Norell JE, Dyck PJ (1999) Microvasculitis and ischemia in diabetic lumbosacral radiculoplexus neuropathy. Neurology 53(9):2113-2113

12. Gibbons CH, Freeman R (2015) Treatment-induced neuropathy of diabetes: an acute, iatrogenic complication of diabetes. Brain 138(1):43-52

13. Tesfaye S, Chaturvedi N, Eaton SE et al (2005) Vascular risk factors and diabetic neuropathy. NEngl J Med 352(4):341-350

14. Callaghan $B C$, Cheng HT, Stables $C L$, Smith $A L$, Feldman EL (2010) Diabetic neuropathy: clinical manifestations and current treatments. Lancet Neurol 11(6):521-534 
15. Witzel II, Jelinek HF, Khalaf K, Lee $S$, Khandoker $A H_{\text {, }}$ Alsafar $\mathrm{H}$ (2015) Identifying common genetic risk factors of diabetic neuropathies. Front Endocrinol (Lausanne) 6:88. https://doi.org/10.3389/fendo. 2015.00088

16. Mielke JG, Wang YT (2011) Insulin, synaptic function, and opportunities for neuroprotection. Prog Mol Biol TransI Sci 98:133-186

17. Chung SS, Ho EC, Lam KS, Chung SK (2003) Contribution of polyol pathway to diabetesinduced oxidative stress. J Am Soc Nephrol 14:S233-S236

18. Cortese A, ZhuY, RebeloAP etal (2020) Biallelicmutations in SORD cause a common and potentially treatable hereditary neuropathy with implications for diabetes. Nat Genet 52(5):473-481. https://doi. org/10.1038/s41588-020-0615-4

19. Othman A, Bianchi R, Alecu l et al (2015) Lowering plasma 1-deoxysphingolipids improves neuropathy in diabetic rats. Diabetes 64(3):1035-1045

20. Sandireddy R, Yerra VG, Areti A et al (2014) Neuroinflammation and oxidative stress in diabetic neuropathy: futuristic strategies based on these targets. Int J Endocrinol. https://doi.org/10. $1155 / 2014 / 674987$

21. Lupachyk S, Watcho P, Stavniichuk R et al (2013) Endoplasmic reticulum stress plays a key role in the pathogenesis of diabetic peripheral neuropathy. Diabetes 62(3):944-952

22. Domènech-Estévez $E$, Baloui $H$, Repond $C$ et al (2015) Distribution of monocarboxylate transporters in the peripheral nervous system suggests putative roles in lactate shuttling and myelination. JNeurosci 35(10):4151-4156

23. Feldman EL, Nave KA, Jensen TS et al (2017) New horizons in diabetic neuropathy: mechanisms, bioenergetics, and pain. Neuron 93(6):1296-1313

24. Viader A, Sasaki Y, Kim S et al (2013) Aberrant Schwann cell lipid metabolism linked to mitochondrial deficits leads to axon degeneration and neuropathy. Neuron 77(5):886-898

25. Brownlee M (2010) Biochemistry and molecular cell biology of diabetic complications. Nature 414(6865):813

26. Malik RA, Tesfaye S, Thompson SD et al (1993) Endoneurial localisation of microvascular damage in human diabetic neuropathy. Diabetologia 36(5):454-459

27. Mohseni S (2001) Hypoglycemic neuropathy. Acta Neuropathol 102:413-421

28. Reichard P, Nilsson B, Rosenqvist U (1993) The effect of long-term intensified insulin treatment on the development of microvascular complications of diabetes mellitus. N Engl J Med 329(5):304-309

29. Nathan DM (2014) The diabetes control and complications trial/epidemiology of diabetes interventions and complications study at 30 years: overview. Diabetes Care 37(1):9-16

30. Wile DJ, Toth C (2010) Association of metformin, elevated homocysteine, and methylmalonic acid levels and clinically worsened diabetic peripheral neuropathy. Diabetes Care 33(1):156-161

31. Nielsen SF, Nordestgaard BG (2014) Statin use before diabetes diagnosis and risk of microvascular disease: a nationwide nested matched study. Lancet Diabetes Endocrinol 2(11):894-900

32. Finnerup NB, Attal N, Haroutounian S et al (2015) Pharmacotherapy for neuropathic pain in adults: a systematic review and meta-analysis. Lancet Neurol 14(2):162-173

33. Vincent AM, Callaghan BC, Smith AL et al (2011) Diabetic neuropathy: cellular mechanisms as therapeutic targets. Nat Rev Neurol 7(10):573
34. Dohrn MF, Kessler S, Dafotakis M (2020) Die Rolle der diabetischen Neuropathie bei der Genese des Charcot-Fußes. Klin Neurophysiol 51(02):67-72. https://doi.org/10.1055/a-1134-2547

35. Ziegler D, Nowak H, Kempler P et al (2004) Treatment of symptomatic diabetic polyneuropathy with the antioxidant a-lipoic acid: a meta-analysis Diabet Med 21(2):114-121

36. Hu X, Li S, Yang G et al (2014) Efficacy and safety of aldose reductase inhibitor for the treatment of diabetic cardiovascular autonomic neuropathy: systematic review and meta-analysis. PLoS One 9(2):e87096 